\title{
Finite-time synchronization of delayed complex dynamical network via pinning control
}

\author{
Feng Xiao and Qintao Gan*
}

"Correspondence:

ganqintao@sina.com

Department of Basic Science,

Shijiazhuang Mechanical

Engineering College, Shijiazhuang,

050003, P.R. China

\section{然 Springer}

\begin{abstract}
We investigate a finite-time synchronization problem of hybrid-coupled delayed dynamical network via pinning control. According to linear feedback principle and finite-time control theory, the finite-time synchronization can be achieved by pinning control with suitable continuous finite-time controller. Some sufficient conditions are given for finite-time synchronization of undirected and directed complex network by applying finite-time stability lemma. Numerical simulations are finally presented to demonstrate the effectiveness of the theoretical results.
\end{abstract}

Keywords: undirected and directed complex network; finite time; pinning synchronization; time delay

\section{Introduction}

Complex networks are commendably in a position to describe many complex models of connection by natural science, social science, management science, engineering technology, and other fields. It depends on mathematics, statistical physics, computer, and other science as tools and aims at complex systems. Therefore, complex networks are an interdiscipline that develops rapidly in the 21st century [1]. In the past decades, with the gradual deepening research of the theory and application of complex networks, people start trying to investigate different large complex systems in the real world by this novel theory tools [1-3], such as Internet, ecological systems, biological neural networks, social networks, and world wide web. Synchronization is a kind of phenomena that exists extensively in the nature and human society. In the meantime, it is one of dynamic behaviors of complex networks. Nowadays, synchronization of complex networks has been an significant research orientation in the network science [2].

In recent years, the synchronization problem of complex networks has attracted more and more attention from different areas, such as mathematics, physical science, communication, engineering, and so on. There are some control schemes to accomplish the synchronization of a complex network, such as pinning control [4-7], adaptive control [8, 9], impulsive control [10,11], and the like. Pinning control is one of the effective methods in the synchronization control of a complex network, and this way is simple, practical, and economic. Because pinning controllers are applied to merely need a small part of nodes to

(c) The Author(s) 2017. This article is distributed under the terms of the Creative Commons Attribution 4.0 International License (http://creativecommons.org/licenses/by/4.0/), which permits unrestricted use, distribution, and reproduction in any medium, provided you give appropriate credit to the original author(s) and the source, provide a link to the Creative Commons license, and indicate if changes were made. 
drive the system in order to achieve the synchronization of complex network, and it is impossible to achieve synchronization by controlling all nodes in the reality. In the pinning control of a complex network, it is common knowledge that one of the most challenging problems is how to select pinned nodes to guarantee the convergence of the pinning process. In [5], the authors have proved that a class of linear coupled complex networks can achieve synchronization when the coupling strength is sufficiently large by pinning a part of nodes.

As everyone knows, time delay is a very common phenomenon in real complex systems. On account of the speeds of transmission, spreading, and traffic congestion, a signal traveling through a complex network is often united with time delay. That is, there is some information communication of nodes not only at time $t$ but also at time $t-\tau$. In realistic situations, moreover, there exists much more complex and uncertain information communication in complex networks, and time delay is not simple just like $x_{i}(t-\tau)$ but much more like $x_{i}\left(t-\tau_{1}(t)\right), x_{i}\left(t-\tau_{2}(t)\right)$, and $x_{i}\left(t-\tau_{3}(t)\right)$. They are called multitime delay and time-varying delay. Consequently, it is necessary to investigate the influence of time delay on the synchronization of a complex network. In some of the previous literature, such as Refs. [5-7, 10, 12], they established some complex models with either no time delay or only single time delay $x_{i}(t-\tau)$, which is significantly different from complex systems of real world.

Throughout this paper, the network model comes from [4], where pinning synchronization of directed delayed dynamical networks via intermittent control was investigated. There are two types of time delay, coupling delay and internal delay. Furthermore, coupling delay is caused by exchange of information between nodes, and internal delay occurs inside the system. In addition, the coupling terms are including transmission delay and self-feedback delay. To our best knowledge, delay is likely to influence the own state of a node and neighboring state. In realistic situations, the transmission delay is different from the self-feedback delay. In [4], the coupling term is written as $\Gamma\left(x_{j}(t-\tau(t))-x_{i}(t-\sigma(t))\right)$. Therefore, both the coupling and internal delays should be taken into account in a realistic system of complex network. However, the synchronization of a complex network with time delay is often achieved for finite time $[10,11,13-17]$ in practical applications. In other words, people are looking forward to achieving the synchronization of a complex network as fast as possible in reality, and the finite-time synchronization means the improved convergence rate in a complex network. It is significant to investigate the finite-time synchronization of a complex network in practice. Nevertheless, in [4], the asymptotic stability or exponential stability of the synchronization error is just investigated. Hence, the main purpose of this paper is to achieve the finite-time synchronization of a complex network with multitime delay and time-varying delay by pinning control. Furthermore, in the paper, the finite-time synchronization problem is described from two aspects, undirected network [18] and directed network $[4,19]$.

Motivated by the aforementioned discussions, the main objective of this paper is to investigate the finite-time synchronization problem of hybrid-coupled complex dynamical networks with different feedback delays and internal delays via using pinning control. By adding finite-time pinning controllers to partial nodes and building a novel differential inequality, some sufficient conditions are derived to ensure the finite-time synchronization of complex dynamical networks. The minimal number of pinned nodes can be identified 
from the proposed conditions. Numerical simulations are described to display the effectiveness of the theoretical results.

The paper is structured as follows. Section 2 provides some mathematical preliminaries and formulates a model with a finite-time synchronization of a delayed complex network. In Section 3, we give some sufficient finite-time synchronization conditions and propose pinned-node schemes that include undirected network and directed network. Numerical simulations are given in Section 4 to verify the effectiveness of the theoretical results. Finally, the paper is concluded in Section 5.

\section{Problem description}

In this section, we consider a complex network consisting of $N$ nodes with each being an $n$-dimensional dynamical system. The complex network is described by

$$
\begin{aligned}
\dot{x}_{i}(t)= & f\left(t, x_{i}(t), x_{i}\left(t-\tau_{1}(t)\right)\right)+c \sum_{j=1, j \neq i}^{N} b_{i j}^{0} \Gamma\left(x_{j}(t)-x_{i}(t)\right) \\
& +c_{\tau} \sum_{j=1, j \neq i}^{N} b_{i j}^{1} \Gamma_{\tau}\left(x_{j}\left(t-\tau_{2}(t)\right)-x_{i}\left(t-\tau_{3}(t)\right)\right),
\end{aligned}
$$

where $i=1,2, \ldots, N, x_{i}(t)=\left(x_{i 1}(t), x_{i 2}(t), \ldots, x_{\text {in }}(t)\right)^{T} \in \mathbf{R}^{n}$ is the state vector of node $i$, $f: \mathbf{R} \times \mathbf{R}^{n} \times \mathbf{R}^{n} \rightarrow \mathbf{R}^{n}$ is a continuous vector-valued function controlling the dynamics of isolated nodes, the time-varying delays $\tau_{1}(t), \tau_{2}(t)$, and $\tau_{3}(t)$ are bounded by known constants, that is, $0 \leq \tau_{1}(t) \leq \tau_{1}, 0 \leq \tau_{2}(t) \leq \tau_{2}$, and $0 \leq \tau_{3}(t) \leq \tau_{3}$, in which $\tau_{1}(t)$ denotes the internal delay occurring inside the nodes, $\tau_{2}(t)$ represents the transmission delay for signal sent from node $j$ to node $i$, and $\tau_{3}(t)$ is the self-feedback delay. The positive constants $c$ and $c_{\tau}$ are the coupling strengths, $\Gamma=\left(\gamma_{i j}\right)_{n \times n}$ and $\Gamma_{\tau}=\left(\gamma_{i j}^{\tau}\right)_{n \times n}$ are the inner connecting matrices, and $B^{0}=\left(b_{i j}^{0}\right)_{N \times N}$ and $B^{1}=\left(b_{i j}^{1}\right)_{N \times N}$ are the outer coupling matrices between the nodes. The most difference between undirected and directed networks is their outer coupling matrices.

In the undirected network, the outer coupling matrices are defined to satisfy the following conditions:

If there exists a connection between nodes $i$ and $j$, then $b_{i j}^{k}=b_{j i}^{k}>0$, and otherwise $b_{i j}^{k}=$ $b_{j i}^{k}=0(i \neq j), k=0,1$. Moreover, $b_{i i}^{k}=-\sum_{j=1, j \neq i} b_{i j}^{k}, k=0,1$.

In the directed network, the outer coupling matrices are defined to satisfy the following conditions:

$$
b_{i j}^{k} \geq 0, \quad i \neq j, \quad b_{i i}^{k}=-\sum_{j=1, j \neq i} b_{i j}^{k}, \quad k=0,1 .
$$

Based on the above two conditions, we can rewrite the network model (2.1) as follows:

$$
\begin{aligned}
\dot{x}(t)= & f\left(t, x_{i}(t), x_{i}\left(t-\tau_{1}(t)\right)\right)+c \sum_{j=1}^{N} b_{i j}^{0} \Gamma x_{j}(t)+c_{\tau} \sum_{j=1}^{N} b_{i j}^{1} \Gamma_{\tau} x_{j}\left(t-\tau_{2}(t)\right) \\
& -c_{\tau} b_{i i}^{1} \Gamma_{\tau}\left(x_{i}\left(t-\tau_{2}(t)\right)-x_{i}\left(t-\tau_{3}(t)\right)\right), \quad i=1,2, \ldots, N .
\end{aligned}
$$

Therefore, the finite-time synchronization problem of the complex network (2.2) is described as follows. 
There exists a time $t^{*}>0$ such that, for all $i, j$ and $t \geq t^{*}$, we have $\left\|x_{i}(t)-s(t)\right\|=0$, $i=1,2, \ldots, N$. Here, $s(t)$ may be an equilibrium point, a periodic orbit, or a chaotic orbit. Then, the network (2.2) reaches complete synchronization in finite time, and we have the following synchronized state equation:

$$
\dot{s}(t)=f\left(t, s(t), s\left(t-\tau_{1}(t)\right)\right)-c_{\tau} b_{i i}^{1} \Gamma_{\tau}\left(s\left(t-\tau_{2}(t)\right)-s\left(t-\tau_{3}(t)\right)\right), \quad i=1,2, \ldots, N
$$

Obviously, the synchronized state $s(t)$ is uniform. Hence, to achieve the complete synchronization of network (2.2) in finite-time, let $b_{11}^{1}=b_{22}^{1}=\cdots=b_{N N}^{1}=-a<0$. Under this condition, the synchronized state equation (2.3) can be written as

$$
\dot{s}(t)=f\left(t, s(t), s\left(t-\tau_{1}(t)\right)\right)+a c_{\tau} \Gamma_{\tau}\left(s\left(t-\tau_{2}(t)\right)-s\left(t-\tau_{3}(t)\right)\right) .
$$

In this paper, to achieve synchronization in finite time, we take advantage of the scheme of pinning control $[15,19-21]$, finite-time controllers $u_{i}(t)$, and synchronized solution $s(t)$ of complex network (2.2), that is,

$$
\lim _{t \rightarrow t^{*}}\left\|x_{i}(t)-s(t)\right\|=0, \quad i=1,2, \ldots, N
$$

To reach the control target, some controllers are added to partial nodes of network (2.2). Without loss of generality, let the first $l(1 \leq l<N)$ nodes to be selected and pinned. Then we have the following controlled delayed network:

$$
\left\{\begin{aligned}
\dot{x}_{i}(t)= & f\left(t, x_{i}(t), x_{i}\left(t-\tau_{1}(t)\right)\right)+a c_{\tau} \Gamma_{\tau}\left(x_{i}\left(t-\tau_{2}(t)\right)-x_{i}\left(t-\tau_{3}(t)\right)\right) \\
& +c \sum_{j=1}^{N} b_{i j}^{0} \Gamma x_{j}(t)+c_{\tau} \sum_{j=1}^{N} b_{i j}^{1} \Gamma_{\tau} x_{j}\left(t-\tau_{2}(t)\right)+u_{i}(t), \quad 1 \leq i \leq l, \\
\dot{x}_{i}(t)= & f\left(t, x_{i}(t), x_{i}\left(t-\tau_{1}(t)\right)\right)+a c_{\tau} \Gamma_{\tau}\left(x_{i}\left(t-\tau_{2}(t)\right)-x_{i}\left(t-\tau_{3}(t)\right)\right) \\
& +c \sum_{j=1}^{N} b_{i j}^{0} \Gamma x_{j}(t)+c_{\tau} \sum_{j=1}^{N} b_{i j}^{1} \Gamma_{\tau} x_{j}\left(t-\tau_{2}(t)\right), \quad l+1 \leq i \leq N,
\end{aligned}\right.
$$

where $u_{i}(t)$ are controllers defined as follows:

$$
\begin{aligned}
u_{i}(t)= & -d_{i} e_{i}(t)-k^{\prime}\left(k_{1} \sum_{i=1}^{N} \int_{t-\tau_{1}(t)}^{t} e_{i}^{\mathrm{T}}(s) e_{i}(s) d s\right)^{\frac{1}{2}} \frac{e_{i}(t)}{\|e(t)\|^{2}} \\
& -k^{\prime} \operatorname{sign}\left(e_{i}(t)\right)-k^{\prime}\left(k_{2} \sum_{i=1}^{N} \int_{t-\tau_{2}(t)}^{t} e_{i}^{\mathrm{T}}(s) e_{i}(s) d s\right)^{\frac{1}{2}} \frac{e_{i}(t)}{\|e(t)\|^{2}} \\
& -k^{\prime}\left(k_{3} \sum_{i=1}^{N} \int_{t-\tau_{3}(t)}^{t} e_{i}^{\mathrm{T}}(s) e_{i}(s) d s\right)^{\frac{1}{2}} \frac{e_{i}(t)}{\|e(t)\|^{2}}, \quad 1 \leq i \leq l,
\end{aligned}
$$

where, $d_{i}>0$ is a positive constant called the control gain, $k^{\prime}, k_{1}, k_{2}$, and $k_{3}$ are positive constants. Let $e_{i}(t)=x_{i}(t)-s(t), 1 \leq i \leq N$, be synchronization errors. Let $\hat{B}^{0 s}=\left(\hat{B}^{0}+\right.$ $\left.\hat{B}^{0 T}\right) / 2$, where $\hat{B}^{0}$ is a modified matrix of $B^{0}$ via replacing the diagonal elements $b_{i i}^{0}$ by $\left(\rho_{\min } / \rho_{0}\right) b_{i i}^{0}$; here $\rho_{\min }$ is the minimum eigenvalue of the matrix $\left(\Gamma+\Gamma^{T}\right) / 2$. By $I_{N}$ we denote the $n$-dimensional identity matrix. Assume that $\rho_{\min }>0$ and $\|\Gamma\|=\rho_{0}>0$. On the basis 
of the control laws (2.7), the error system is governed as follows:

$$
\left\{\begin{aligned}
\dot{e}_{i}(t)=\tilde{f}\left(t, x_{i}, s, x_{i}^{\tau_{1}}, s^{\tau_{1}}\right)+a c_{\tau} \Gamma_{\tau}\left(e_{i}\left(t-\tau_{2}(t)\right)-e_{i}\left(t-\tau_{3}(t)\right)\right)+c \sum_{j=1}^{N} b_{i j}^{0} \Gamma e_{j}(t) \\
\quad+c_{\tau} \sum_{j=1}^{N} b_{i j}^{1} \Gamma_{\tau} e_{j}\left(t-\tau_{2}(t)\right)+u_{i}(t), \quad 1 \leq i \leq l, \\
\dot{e}_{i}(t)=\tilde{f}\left(t, x_{i}, s, x_{i}^{\tau_{1}}, s^{\tau_{1}}\right)+a c_{\tau} \Gamma_{\tau}\left(e_{i}\left(t-\tau_{2}(t)\right)-e_{i}\left(t-\tau_{3}(t)\right)\right)+c \sum_{j=1}^{N} b_{i j}^{0} \Gamma e_{j}(t) \\
\quad+c_{\tau} \sum_{j=1}^{N} b_{i j}^{1} \Gamma_{\tau} e_{j}\left(t-\tau_{2}(t)\right), \quad l+1 \leq i \leq N,
\end{aligned}\right.
$$

where $\tilde{f}\left(t, x_{i}, s, x_{i}^{\tau_{1}}, s^{\tau_{1}}\right)=f\left(t, x_{i}(t), x_{i}\left(t-\tau_{1}(t)\right)\right)-f\left(t, s(t), s\left(t-\tau_{1}(t)\right)\right)$.

The following assumptions and lemmas are needed to derive our main results.

Assumption 1 ([6]) The vector-valued function $f\left(t, x_{i}(t), x_{i}\left(t-\tau_{1}(t)\right)\right)$ satisfies the uniform semi-Lipschitz condition with respect time $t$, that is, for any $x(t), y(t) \in R^{n}$, there exist positive constants $L_{1}>0$ and $L_{2}>0$ such that

$$
\begin{aligned}
(x(t) & -y(t))^{T}\left(f\left(t, x(t), x\left(t-\tau_{1}(t)\right)\right)-f\left(t, y(t), y\left(t-\tau_{1}(t)\right)\right)\right) \\
\leq & L_{1}(x(t)-y(t))^{T}(x(t)-y(t))+L_{2}\left(x\left(t-\tau_{1}(t)\right)-y\left(t-\tau_{1}(t)\right)\right)^{T} \\
& \times\left(x\left(t-\tau_{1}(t)\right)-y\left(t-\tau_{1}(t)\right)\right) .
\end{aligned}
$$

Assumption 2 The time-varying delays $\tau_{i}(t)(i=1,2,3)$ are differentiable functions, and $0 \leq \dot{\tau}_{i}(t) \leq \psi_{i} \leq 1$, where $\psi_{i}(i=1,2,3)$ are constants.

Lemma 1 ([4]) If $Y$ and $Z$ are real matrices of appropriate dimensions, then there exists a positive constant $\xi>0$ such that

$$
Y^{T} Z+Z^{T} Y \leq \xi Y^{T} Y+\frac{1}{\xi} Z^{T} Z
$$

Lemma 2 ([1]) Assume that $Q=\left(q_{i j}\right)_{N \times N}$ is symmetric. Let $D=\operatorname{diag}(d_{1}, \ldots, d_{l}, \overbrace{0, \ldots, 0}^{N-l})$, $Q-D=\left(\begin{array}{cc}E-\tilde{D} & S \\ S^{T} & Q_{l}\end{array}\right)$, and $d=\min _{1 \leq i<l}\left\{d_{i}\right\}$, where $1 \leq l<N, d_{i}>0, i=1, \ldots, l, Q_{l}$ is the minor matrix of $Q$ by removing its first $l(1 \leq l<N)$ row-column pairs, $E$ and $S$ are matrices of appropriate dimensions, $\tilde{D}=\operatorname{diag}\left(d_{1}, \ldots, d_{l}\right)$. If $d>\lambda_{\max }\left(E-S Q_{l}^{-1} S^{T}\right)$, then $Q-D<0$ is equivalent to $Q_{l}<0$.

Lemma 3 ([10]) For $x_{1}, x_{2}, \ldots, x_{n} \in R^{n}$,

$$
\left\|x_{1}\right\|+\left\|x_{2}\right\|+\cdots+\left\|x_{n}\right\| \geq \sqrt{\left\|x_{1}\right\|^{2}+\left\|x_{2}\right\|^{2}+\cdots+\left\|x_{n}\right\|^{2}} .
$$

Lemma $4([11,15,16])$ Suppose that a continuous and positive definite function $V(t)$ satisfies the inequality

$$
\dot{V}(t) \leq-p V^{\xi}(t), \quad \forall t \geq t_{0}, \quad V\left(t_{0}\right) \geq 0,
$$

where $p>0$ and $0<\xi<1$ are two constants. For any given time $t_{0}, V(t)$ satisfies the following inequality: $V^{1-\xi}(t) \leq V^{1-\xi}\left(t_{0}\right)-p(1-\xi)\left(t-t_{0}\right), t_{0} \leq t \leq t_{1}$, and $V(t) \equiv 0$ for all $t \geq t_{1}$ with $t_{1}$ given by $t_{1}=t_{0}+V^{1-\xi}\left(t_{0}\right) /[p(1-\xi)]$. 


\section{Main results}

\subsection{Finite-time synchronization problem of undirected network}

Theorem 1 Suppose that Assumption 1 holds. Suppose that there exist positive constants $\varsigma_{1}, \varsigma_{2}, \varsigma_{3}, q$, and $d_{i}, i=1, \ldots, l$, such that

(1) $(p+q) I_{N}+c \rho_{0} \hat{B}^{0}-D<0$

(2) $L_{2}-\frac{1-\psi_{1}}{2} k_{1}<0$,

(3) $a c_{\tau} \frac{\lambda_{\max }\left(\Gamma_{\tau}^{\mathrm{T}} \Gamma_{\tau}\right)}{2 \varsigma_{1}}+\frac{1}{2 \varsigma_{3}}-\frac{1-\psi_{2}}{2} k_{2}<0$,

(4) $a c_{\tau} \frac{\lambda_{\max }\left(\Gamma_{\tau}^{\mathrm{T}} \Gamma_{\tau}\right)}{2 \varsigma_{2}}-\frac{1-\psi_{3}}{2} k_{3}<0$,

(5) $t_{1}^{*}=t_{0}+\frac{\sqrt{2}}{k^{\prime}} V^{\frac{1}{2}}\left(t_{0}\right)$

where $V\left(t_{0}\right)=\frac{1}{2} \sum_{i=1}^{N} e_{i}^{T}\left(t_{0}\right) e_{i}\left(t_{0}\right)+\frac{1}{2} k_{1} \sum_{i=1}^{N} \int_{t_{0}-\tau_{1}\left(t_{0}\right)}^{t_{0}} e_{i}^{\mathrm{T}}(s) e_{i}(s) d s+\frac{1}{2} k_{2} \sum_{i=1}^{N} \int_{t_{0}-\tau_{2}\left(t_{0}\right)}^{t_{0}} e_{i}^{\mathrm{T}}(s) \times$ $e_{i}(s) d s+\frac{1}{2} k_{3} \sum_{i=1}^{N} \int_{t_{0}-\tau_{3}\left(t_{0}\right)}^{t_{0}} e_{i}^{\mathrm{T}}(s) e_{i}(s) d s, e_{i}\left(t_{0}\right)$ and $\tau_{i}\left(t_{0}\right)(i=1,2,3)$ are initial conditions of $e_{i}(t)$ and $\tau_{i}(t), p=L_{1}+a c_{\tau} \frac{\varsigma 1}{2}+a c_{\tau} \frac{\varsigma 2}{2}+\frac{\varsigma 3}{2} \lambda_{\max }\left(B^{1} B^{1 T}\right) \lambda_{\max }\left(\Gamma_{\tau} \Gamma_{\tau}^{\mathrm{T}}\right), D=\operatorname{diag}\left(d_{1}, \ldots, d_{l}\right.$, $N-l$

$\overbrace{0, \ldots, 0})$, and $q=\frac{1}{2} k_{1}+\frac{1}{2} k_{2}+\frac{1}{2} k_{3}$. Then the complex network (2.6) can achieve synchronization in finite-time $t \leq t_{1}^{*}$.

Proof Construct a Lyapunov-Krasovskii functional as follows:

$$
\begin{aligned}
V(t)= & \frac{1}{2} \sum_{i=1}^{N} e_{i}^{T}(t) e_{i}(t)+\frac{1}{2} k_{1} \sum_{i=1}^{N} \int_{t-\tau_{1}(t)}^{t} e_{i}^{\mathrm{T}}(s) e_{i}(s) d s \\
& +\frac{1}{2} k_{2} \sum_{i=1}^{N} \int_{t-\tau_{2}(t)}^{t} e_{i}^{\mathrm{T}}(s) e_{i}(s) d s+\frac{1}{2} k_{3} \sum_{i=1}^{N} \int_{t-\tau_{3}(t)}^{t} e_{i}^{\mathrm{T}}(s) e_{i}(s) d s .
\end{aligned}
$$

Taking the derivative of $V(t)$ with respect to time $t$ along the solution of network (2.6), we obtain

$$
\begin{aligned}
\dot{V}(t)= & \sum_{i=1}^{N} e_{i}^{\mathrm{T}}(t)\left[\tilde{f}\left(t, x_{i}, s, x_{i}^{\tau_{1}}, s^{\tau_{1}}\right)+a c_{\tau} \Gamma_{\tau}\left(e_{i}\left(t-\tau_{2}(t)\right)-e_{i}\left(t-\tau_{3}(t)\right)\right)\right. \\
& \left.+c \sum_{j=1}^{N} b_{i j}^{0} \Gamma e_{j}(t)+c_{\tau} \sum_{j=1}^{N} b_{i j}^{1} \Gamma_{\tau} e_{j}\left(t-\tau_{2}(t)\right)+u_{i}(t)\right] \\
& -\frac{1-\dot{\tau}_{1}(t)}{2} k_{1} \sum_{i=1}^{N} e_{i}^{\mathrm{T}}\left(t-\tau_{1}(t)\right) e_{i}\left(t-\tau_{1}(t)\right)+\frac{1}{2} k_{1} \sum_{i=1}^{N} e_{i}^{\mathrm{T}}(t) e_{i}(t) \\
& -\frac{1-\dot{\tau}_{2}(t)}{2} k_{2} \sum_{i=1}^{N} e_{i}^{\mathrm{T}}\left(t-\tau_{2}(t)\right) e_{i}\left(t-\tau_{2}(t)\right)+\frac{1}{2} k_{2} \sum_{i=1}^{N} e_{i}^{\mathrm{T}}(t) e_{i}(t) \\
& -\frac{1-\dot{\tau}_{3}(t)}{2} k_{3} \sum_{i=1}^{N} e_{i}^{\mathrm{T}}\left(t-\tau_{3}(t)\right) e_{i}\left(t-\tau_{3}(t)\right)+\frac{1}{2} k_{3} \sum_{i=1}^{N} e_{i}^{\mathrm{T}}(t) e_{i}(t) .
\end{aligned}
$$

Using Assumption 1, we have

$$
\sum_{i=1}^{N} e_{i}^{T}(t) \tilde{f}\left(t, x_{i}, s, x_{i}^{\tau_{1}}, s^{\tau_{1}}\right) \leq L_{1} \sum_{i=1}^{N} e_{i}^{T}(t) e_{i}(t)+L_{2} \sum_{i=1}^{N} e_{i}^{T}\left(t-\tau_{1}(t)\right) e_{i}\left(t-\tau_{1}(t)\right)
$$


Then we get

$$
\begin{aligned}
\dot{V}(t) \leq & L_{1} \sum_{i=1}^{N} e_{i}^{\mathrm{T}}(t) e_{i}(t)+L_{2} \sum_{i=1}^{N} e_{i}^{\mathrm{T}}\left(t-\tau_{1}(t)\right) e_{i}\left(t-\tau_{1}(t)\right)+a c_{\tau} \sum_{i=1}^{N} e_{i}^{\mathrm{T}}(t) \Gamma_{\tau} e_{i}\left(t-\tau_{2}(t)\right) \\
& -a c_{\tau} \sum_{i=1}^{N} e_{i}^{\mathrm{T}}(t) \Gamma_{\tau} e_{i}\left(t-\tau_{3}(t)\right)+c \sum_{i=1}^{N} \sum_{j=1, j \neq i}^{N} b_{i j}^{0} e_{i}^{\mathrm{T}}(t) \Gamma e_{j}(t)+c \sum_{i=1}^{N} b_{i i}^{0} \rho_{\min } e_{i}^{T}(t) e_{i}(t) \\
& +c_{\tau} \sum_{i=1}^{N} \sum_{j=1}^{N} b_{i j}^{1} e_{i}^{\mathrm{T}}(t) \Gamma_{\tau} e_{j}\left(t-\tau_{2}(t)\right)+\sum_{i=1}^{N} e_{i}^{\mathrm{T}}(t) u_{i}(t)+\frac{1}{2} k_{1} \sum_{i=1}^{N} e_{i}^{\mathrm{T}}(t) e_{i}(t) \\
& -\frac{1-\dot{\tau}_{1}(t)}{2} k_{1} \sum_{i=1}^{N} e_{i}^{\mathrm{T}}\left(t-\tau_{1}(t)\right) e_{i}\left(t-\tau_{1}(t)\right) \\
& -\frac{1-\dot{\tau}_{2}(t)}{2} k_{2} \sum_{i=1}^{N} e_{i}^{\mathrm{T}}\left(t-\tau_{2}(t)\right) e_{i}\left(t-\tau_{2}(t)\right) \\
& +\frac{1}{2} k_{2} \sum_{i=1}^{N} e_{i}^{\mathrm{T}}(t) e_{i}(t)+\frac{1}{2} k_{3} \sum_{i=1}^{N} e_{i}^{\mathrm{T}}(t) e_{i}(t) \\
& -\frac{1-\dot{\tau}_{3}(t)}{2} k_{3} \sum_{i=1}^{N} e_{i}^{\mathrm{T}}\left(t-\tau_{3}(t)\right) e_{i}\left(t-\tau_{3}(t)\right) .
\end{aligned}
$$

From Lemma 1 and the property of the Kronecker product of the matrices we derive the following inequalities:

$$
\begin{aligned}
& a c_{\tau} \sum_{i=1}^{N} e_{i}^{T}(t) \Gamma_{\tau} e_{i}\left(t-\tau_{2}(t)\right) \\
& \leq \frac{a c_{\tau}}{2}\left[\varsigma_{1} \sum_{i=1}^{N} e_{i}^{T}(t) e_{i}(t)+\frac{1}{\varsigma_{1}} \sum_{i=1}^{N} e_{i}^{T}\left(t-\tau_{2}(t)\right) \Gamma_{\tau}^{T} \Gamma_{\tau} e_{i}\left(t-\tau_{2}(t)\right)\right] \\
& \leq a c_{\tau} \frac{\varsigma_{1}}{2} \sum_{i=1}^{N} e_{i}^{T}(t) e_{i}(t)+a c_{\tau} \frac{\lambda_{\max }\left(\Gamma_{\tau}^{T} \Gamma_{\tau}\right)}{2 \varsigma_{1}} \sum_{i=1}^{N} e_{i}^{T}\left(t-\tau_{2}(t)\right) e_{i}\left(t-\tau_{2}(t)\right) \\
& -a c_{\tau} \sum_{i=1}^{N} e_{i}^{T}(t) \Gamma_{\tau} e_{i}\left(t-\tau_{3}(t)\right) \\
& \leq \frac{a c_{\tau}}{2}\left[\varsigma_{2} \sum_{i=1}^{N} e_{i}^{T}(t) e_{i}(t)+\frac{1}{\varsigma_{2}} \sum_{i=1}^{N} e_{i}^{T}\left(t-\tau_{3}(t)\right) \Gamma_{\tau}^{T} \Gamma_{\tau} e_{i}\left(t-\tau_{3}(t)\right)\right] \\
& \leq a c_{\tau} \frac{\varsigma_{2}}{2} \sum_{i=1}^{N} e_{i}^{T}(t) e_{i}(t)+a c_{\tau} \frac{\lambda_{\max }\left(\Gamma_{\tau}^{T} \Gamma_{\tau}\right)}{2 \varsigma_{2}} \sum_{i=1}^{N} e_{i}^{T}\left(t-\tau_{3}(t)\right) e_{i}\left(t-\tau_{3}(t)\right) \\
& \sum_{i=1}^{N} \sum_{j=1}^{N} b_{i j}^{1} e_{i}^{T}(t) \Gamma_{\tau} e_{j}\left(t-\tau_{2}(t)\right) \\
& \quad=e^{T}(t)\left(B^{1} \otimes \Gamma_{\tau}\right) e\left(t-\tau_{2}(t)\right) \\
& \leq \frac{1}{2}\left[\varsigma_{3} e^{T}(t)\left(B^{1} B^{1 T} \otimes \Gamma_{\tau} \Gamma_{\tau}^{T}\right) e(t)+\frac{1}{\varsigma_{3}} e^{T}\left(t-\tau_{2}(t)\right)\left(I_{N} \otimes I_{n}\right) e\left(t-\tau_{2}(t)\right)\right]
\end{aligned}
$$




$$
\begin{aligned}
\leq & \frac{\varsigma_{3}}{2} \lambda_{\max }\left(B^{1} B^{1 T}\right) \lambda_{\max }\left(\Gamma_{\tau} \Gamma_{\tau}^{\mathrm{T}}\right) \sum_{i=1}^{N} e_{i}^{T}(t) e_{i}(t) \\
& +\frac{1}{2 \varsigma_{3}} \sum_{i=1}^{N} e_{i}^{T}\left(t-\tau_{2}(t)\right) e_{i}\left(t-\tau_{2}(t)\right) .
\end{aligned}
$$

Substituting (3.5)-(3.7) into (3.4) gives

$$
\begin{aligned}
& \dot{V}(t) \leq L_{1} \sum_{i=1}^{N} e_{i}^{\mathrm{T}}(t) e_{i}(t)+L_{2} \sum_{i=1}^{N} e_{i}^{\mathrm{T}}\left(t-\tau_{1}(t)\right) e_{i}\left(t-\tau_{1}(t)\right) \\
& +a c_{\tau} \frac{\lambda_{\max }\left(\Gamma_{\tau}^{\mathrm{T}} \Gamma_{\tau}\right)}{2 \zeta_{1}} \sum_{i=1}^{N} e_{i}^{\mathrm{T}}\left(t-\tau_{2}(t)\right) e_{i}\left(t-\tau_{2}(t)\right)+a c_{\tau} \frac{\varsigma_{1}}{2} \sum_{i=1}^{N} e_{i}^{\mathrm{T}}(t) e_{i}(t) \\
& +a c_{\tau} \frac{\zeta_{2}}{2} \sum_{i=1}^{N} e_{i}^{\mathrm{T}}(t) e_{i}(t)+a c_{\tau} \frac{\lambda_{\max }\left(\Gamma_{\tau}^{\mathrm{T}} \Gamma_{\tau}\right)}{2 \varsigma_{2}} \sum_{i=1}^{N} e_{i}^{T}\left(t-\tau_{3}(t)\right) e_{i}\left(t-\tau_{3}(t)\right) \\
& +c \sum_{i=1}^{N} \sum_{j=1, j \neq i}^{N} b_{i j}^{0} \rho_{0}\left\|e^{\mathrm{T}}(t)\right\|\left\|e_{j}(t)\right\|+c \sum_{i=1}^{N} b_{i i}^{0} \rho_{\min } e_{i}^{T}(t) e_{i}(t) \\
& +\frac{\varsigma_{3}}{2} \lambda_{\max }\left(B^{1} B^{1 T}\right) \lambda_{\max }\left(\Gamma_{\tau} \Gamma_{\tau}^{\mathrm{T}}\right) \sum_{i=1}^{N} e_{i}^{\mathrm{T}}(t) e_{i}(t) \\
& +\frac{1}{2 \varsigma_{3}} \sum_{i=1}^{N} e_{i}^{\mathrm{T}}\left(t-\tau_{2}(t)\right) e_{i}\left(t-\tau_{2}(t)\right) \\
& -\frac{1-\dot{\tau}_{1}(t)}{2} k_{1} \sum_{i=1}^{N} e_{i}^{\mathrm{T}}\left(t-\tau_{1}(t)\right) e_{i}\left(t-\tau_{1}(t)\right) \\
& -\frac{1-\dot{\tau}_{2}(t)}{2} k_{2} \sum_{i=1}^{N} e_{i}^{\mathrm{T}}\left(t-\tau_{2}(t)\right) e_{i}\left(t-\tau_{2}(t)\right) \\
& -\frac{1-\dot{\tau}_{3}(t)}{2} k_{3} \sum_{i=1}^{N} e_{i}^{\mathrm{T}}\left(t-\tau_{3}(t)\right) e_{i}\left(t-\tau_{3}(t)\right)+q \sum_{i=1}^{N} e_{i}^{\mathrm{T}}(t) e_{i}(t) \\
& -k^{\prime} \sum_{i=1}^{N} e_{i}^{\mathrm{T}}(t)\left[d_{i} e_{i}(t)+\operatorname{sign}\left(e_{i}(t)\right)+\left(k_{1} \sum_{i=1}^{N} \int_{t-\tau_{1}(t)}^{t} e_{i}^{\mathrm{T}}(s) e_{i}(s) d s\right)^{\frac{1}{2}} \frac{e_{i}(t)}{\|e(t)\|^{2}}\right. \\
& +\left(k_{2} \sum_{i=1}^{N} \int_{t-\tau_{2}(t)}^{t} e_{i}^{\mathrm{T}}(s) e_{i}(s) d s\right)^{\frac{1}{2}} \frac{e_{i}(t)}{\|e(t)\|^{2}} \\
& \left.+\left(k_{3} \sum_{i=1}^{N} \int_{t-\tau_{3}(t)}^{t} e_{i}^{\mathrm{T}}(s) e_{i}(s) d s\right)^{\frac{1}{2}} \frac{e_{i}(t)}{\|e(t)\|^{2}}\right] .
\end{aligned}
$$

Letting $e(t)=\left(e_{1}^{\mathrm{T}}(t), e_{2}^{\mathrm{T}}(t), \ldots, e_{N}^{\mathrm{T}}(t)\right)^{\mathrm{T}}$, we obtain

$$
\frac{1}{2} e^{T}(t) e(t)=\frac{1}{2} \sum_{i=1}^{N}\left\|e_{i}(t)\right\|^{2}=\frac{1}{2} \sum_{i=1}^{N} e_{i}^{T}(t) e_{i}(t)=V(t) .
$$


Then, from (3.8) we have

$$
\begin{aligned}
& \dot{V}(t) \leq L_{1} e^{\mathrm{T}}(t) e(t)+L_{2} e^{\mathrm{T}}\left(t-\tau_{1}(t)\right) e\left(t-\tau_{1}(t)\right)+a c_{\tau} \frac{\varsigma_{1}}{2} e^{\mathrm{T}}(t) e(t) \\
& +a c_{\tau} \frac{\lambda_{\max }\left(\Gamma_{\tau}^{\mathrm{T}} \Gamma_{\tau}\right)}{2 \varsigma_{1}} e^{\mathrm{T}}\left(t-\tau_{2}(t)\right) e\left(t-\tau_{2}(t)\right)+a c_{\tau} \frac{\varsigma_{2}}{2} e^{\mathrm{T}}(t) e(t) \\
& +a c_{\tau} \frac{\lambda_{\max }\left(\Gamma_{\tau}^{\mathrm{T}} \Gamma_{\tau}\right)}{2 \varsigma_{2}} e^{\mathrm{T}}\left(t-\tau_{3}(t)\right) e\left(t-\tau_{3}(t)\right)+c \rho_{0} e^{\mathrm{T}}(t) \hat{B}^{0} e(t) \\
& +\frac{\varsigma_{3}}{2} \lambda_{\max }\left(B^{1} B^{1 T}\right) \lambda_{\max }\left(\Gamma_{\tau} \Gamma_{\tau}^{\mathrm{T}}\right) e^{\mathrm{T}}(t) e(t)+\frac{1}{2 \varsigma_{3}} e^{\mathrm{T}}\left(t-\tau_{2}(t)\right) e\left(t-\tau_{2}(t)\right) \\
& -\frac{1-\dot{\tau}_{1}(t)}{2} k_{1} e^{\mathrm{T}}\left(t-\tau_{1}(t)\right) e\left(t-\tau_{1}(t)\right)-\frac{1-\dot{\tau}_{2}(t)}{2} k_{2} e^{\mathrm{T}}\left(t-\tau_{2}(t)\right) e\left(t-\tau_{2}(t)\right) \\
& -\frac{1-\dot{\tau}_{3}(t)}{2} k_{3} e^{\mathrm{T}}\left(t-\tau_{3}(t)\right) e\left(t-\tau_{3}(t)\right)+q \sum_{i=1}^{N} e_{i}^{\mathrm{T}}(t) e_{i}(t)-D e^{\mathrm{T}}(t) e(t) \\
& -k^{\prime} \sum_{i=1}^{N} e_{i}^{\mathrm{T}}(t)\left[\operatorname{sign}\left(e_{i}(t)\right)+\left(k_{1} \sum_{i=1}^{N} \int_{t-\tau_{1}(t)}^{t} e_{i}^{\mathrm{T}}(s) e_{i}(s) d s\right)^{\frac{1}{2}} \frac{e_{i}(t)}{\|e(t)\|^{2}}\right. \\
& +\left(k_{2} \sum_{i=1}^{N} \int_{t-\tau_{2}(t)}^{t} e_{i}^{\mathrm{T}}(s) e_{i}(s) d s\right)^{\frac{1}{2}} \frac{e_{i}(t)}{\|e(t)\|^{2}} \\
& \left.+\left(k_{3} \sum_{i=1}^{N} \int_{t-\tau_{3}(t)}^{t} e_{i}^{\mathrm{T}}(s) e_{i}(s) d s\right)^{\frac{1}{2}} \frac{e_{i}(t)}{\|e(t)\|^{2}}\right] .
\end{aligned}
$$

According to Theorem 1, Assumption 2, and Lemma 3, it follows from (3.10) that

$$
\begin{aligned}
\dot{V}(t) \leq & \left(p+c \rho_{0} \hat{B}^{0}+q-D\right) e^{\mathrm{T}}(t) e(t)+\left(L_{2}-\frac{1-\psi_{1}}{2} k_{1}\right) e^{\mathrm{T}}\left(t-\tau_{1}(t)\right) e\left(t-\tau_{1}(t)\right) \\
& +\left(a c_{\tau} \frac{\lambda_{\max }\left(\Gamma_{\tau}^{\mathrm{T}} \Gamma_{\tau}\right)}{2 \varsigma_{1}}+\frac{1}{2 \varsigma_{3}}-\frac{1-\psi_{2}}{2} k_{2}\right) e^{\mathrm{T}}\left(t-\tau_{2}(t)\right) e\left(t-\tau_{2}(t)\right) \\
& +\left(a c_{\tau} \frac{\lambda_{\max }\left(\Gamma_{\tau}^{\mathrm{T}} \Gamma_{\tau}\right)}{2 \varsigma_{2}}-\frac{1-\psi_{3}}{2} k_{3}\right) e^{\mathrm{T}}\left(t-\tau_{3}(t)\right) e\left(t-\tau_{3}(t)\right) \\
& -k^{\prime} \sum_{i=1}^{N} e_{i}^{\mathrm{T}}(t)\left[\operatorname{sign}\left(e_{i}(t)\right)+\left(k_{1} \sum_{i=1}^{N} \int_{t-\tau_{1}(t)}^{t} e_{i}^{\mathrm{T}}(s) e_{i}(s) d s\right)^{\frac{1}{2}} \frac{e_{i}(t)}{\|e(t)\|^{2}}\right. \\
& +\left(k_{2} \sum_{i=1}^{N} \int_{t-\tau_{2}(t)}^{t} e_{i}^{\mathrm{T}}(s) e_{i}(s) d s\right)^{\frac{1}{2}} \frac{e_{i}(t)}{\|e(t)\|^{2}} \\
& \left.+\left(k_{3} \sum_{i=1}^{N} \int_{t-\tau_{3}(t)}^{t} e_{i}^{\mathrm{T}}(s) e_{i}(s) d s\right)^{\frac{1}{2}} \frac{e_{i}(t)}{\|e(t)\|^{2}}\right] \\
\leq & -k^{\prime} \sum_{i=1}^{N} e_{i}^{\mathrm{T}}(t)\left[\operatorname{sign}\left(e_{i}(t)\right)+\left(k_{1} \sum_{i=1}^{N} \int_{t-\tau_{1}(t)}^{t} e_{i}^{\mathrm{T}}(s) e_{i}(s) d s\right)^{\frac{1}{2}} \frac{e_{i}(t)}{\|e(t)\|^{2}}\right. \\
& +\left(k_{2} \sum_{i=1}^{N} \int_{t-\tau_{2}(t)}^{t} e_{i}^{\mathrm{T}}(s) e_{i}(s) d s\right)^{\frac{1}{2}} \frac{e_{i}(t)}{\|e(t)\|^{2}} \\
& \left({ }_{i}{ }^{2}\right.
\end{aligned}
$$




$$
\begin{aligned}
& \left.+\left(k_{3} \sum_{i=1}^{N} \int_{t-\tau_{3}(t)}^{t} e_{i}^{\mathrm{T}}(s) e_{i}(s) d s\right)^{\frac{1}{2}} \frac{e_{i}(t)}{\|e(t)\|^{2}}\right] \\
\leq & -k^{\prime} \sum_{i=1}^{N} e_{i}^{\mathrm{T}}(t) \frac{e_{i}(t)}{\|e(t)\|^{2}}\left[\left(k_{1} \sum_{i=1}^{N} \int_{t-\tau_{1}(t)}^{t} e_{i}^{\mathrm{T}}(s) e_{i}(s) d s\right)^{\frac{1}{2}}\right. \\
& +\left(k_{2} \sum_{i=1}^{N} \int_{t-\tau_{2}(t)}^{t} e_{i}^{\mathrm{T}}(s) e_{i}(s) d s\right)^{\frac{1}{2}} \\
& \left.+\left(k_{3} \sum_{i=1}^{N} \int_{t-\tau_{3}(t)}^{t} e_{i}^{\mathrm{T}}(s) e_{i}(s) d s\right)^{\frac{1}{2}}+\left\|e_{i}(t)\right\|\right] \\
\leq & -k^{\prime} \sum_{i=1}^{N}\left\|e_{i}(t)\right\|-k^{\prime} \sum_{i=1}^{N}\left(k_{1} \sum_{i=1}^{N} \int_{t-\tau_{1}(t)}^{t} e_{i}^{\mathrm{T}}(s) e_{i}(s) d s\right)^{\frac{1}{2}} \\
& -k^{\prime} \sum_{i=1}^{N}\left(k_{2} \sum_{i=1}^{N} \int_{t-\tau_{2}(t)}^{t} e_{i}^{\mathrm{T}}(s) e_{i}(s) d s\right)^{\frac{1}{2}} \\
& -k^{\prime} \sum_{i=1}^{N}\left(k_{3} \sum_{i=1}^{N} \int_{t-\tau_{3}(t)}^{t} e_{i}^{\mathrm{T}}(s) e_{i}(s) d s\right)^{\frac{1}{2}} \\
\leq & -\sqrt{2} k^{\prime}\left(\frac{1}{2} \sum_{i=1}^{N} e_{i}^{2}(t)+\frac{1}{2} k_{1} \sum_{i=1}^{N} \int_{t-\tau_{1}(t)}^{t} e_{i}^{\mathrm{T}}(s) e_{i}(s) d s\right. \\
+ & \left.-\sqrt{2} k^{\prime} k_{2} \sum_{i=1}^{N} \int_{t-\tau_{2}(t)}^{t} e_{i}^{\mathrm{T}}(t) e_{i}(s) d s+\frac{1}{2} k_{3} \sum_{i=1}^{N} \int_{t-\tau_{3}(t)}^{t} e_{i}^{\mathrm{T}}(s) e_{i}(s) d s\right)^{\frac{1}{2}} \\
& \\
&
\end{aligned}
$$

According to Lemma 4, the complex network (2.1) can achieve synchronization in finite time

$$
t \leq t_{0}+\frac{\sqrt{2}}{k^{\prime}} V^{\frac{1}{2}}\left(t_{0}\right)
$$

Therefore, the proof is completed.

Remark 1 Let $Q=(p+q) I_{N}+c \rho_{0} \hat{B}^{0},(p+q) I_{N}+c \rho_{0} \hat{B}^{0}-D=Q-D=\left(\begin{array}{cc}E-\tilde{D} & S \\ S^{\mathrm{T}} & \mathrm{Q}_{l}\end{array}\right)$, and $d=$ $\min _{1 \leq i \leq l}\left\{d_{i}\right\}$, where $Q_{l}$ is the minor matrix of $Q$ by removing its first $l(1 \leq l<N)$ rowcolumn pairs, $E$ and $S$ are matrices of appropriate dimensions, and $\tilde{D}=\operatorname{diag}\left(d_{1}, \ldots, d_{l}\right)$. By Lemma 2 we know that $Q-D<0$ is equivalent to $Q_{l}<0$ and that $d>\lambda_{\max }\left(E-S Q_{l}^{-1} S^{T}\right)$. If $d$ can be sufficiently large (let $\left.d_{i}>\lambda_{\max }\left(E-S Q_{l}^{-1} S^{T}\right), i=1, \ldots, l\right)$, then $Q-D<0$ is equivalent to $Q_{l}<0$, where $Q_{l}=(p+q) I_{N-l}+c \rho_{0} \hat{B}_{l}^{0}$ with $\hat{B}_{l}^{0}$ the minor matrix of $\hat{B}^{0}$ by removing its first $l(1 \leq l<N)$ row-column pairs.

Corollary 1 We discuss about how to select pinned nodes and how many nodes are pinned at least in an undirected network. Let $C=Q-D=(p+q) I_{N}+c \rho_{\min } B^{0}-D, C=\left(C_{i j}\right)_{N \times N}$. Let $g_{i}=\sum_{j=1, j \neq i}^{N} b_{i j}^{0}$ be the total weights between node $i$ and all other nodes, where $g_{i}$ denotes 
the degree of node $i$. To satisfy $C=Q-D<0$, the necessary conditions are as follows:

$$
\begin{aligned}
& C_{i i}=(p+q)-c \rho_{\min } g_{i}-d_{i}<0, \quad 1 \leq i \leq l, \\
& C_{i i}=(p+q)-c \rho_{\min } g_{i}<0, \quad l+1 \leq i \leq N .
\end{aligned}
$$

From (3.11) we can see that, for the nodes without pinning controllers. it is necessary to make their degrees $g_{i}>\frac{p+q}{c \rho_{\min }}$. When the coupling strength $c$ is very large, the pinning condition is easily to be arrived. In other words, the degree of each node satisfies $C<0$ [6]. But, if $c$ is not very large, then the nodes with low degrees should be controlled by controllers. As we all know, the nodes with low degrees receive a little information from other nodes, which need to be synchronized by controllers because of the nonlinearity of single node. Similarly, the nodes with high degrees receive more information from other nodes, that is, controlling one node with high degrees is equivalent to controlling some or dozens of nodes. Therefore, we should choose two kinds of nodes with low and high degrees in the undirected network. Firstly, we rearrange the nodes by size of degree in descending order. Then, we select pinned nodes from both sides to the middle. In this way, the nodes with low and high degrees can be taken into consideration.

According to Remark 1, for the sake of achieving the synchronization in finite-time, the condition $Q-D<0$ needs to be satisfied, and when $d$ is sufficiently large, $Q-D<0$ is equivalent to $Q_{l}<0$, that is, $(p+q) I_{N-l}+c \rho_{0} \hat{B}_{l}^{0}<0$. We can certainly assume that at least $l_{0}$ nodes need to be pinned so as to accomplish condition $Q_{l}<0$, where $l_{0}$ pinned-nodes satisfy

$$
\lambda_{\max }\left(\hat{B}_{l_{0}-1}^{0}\right) \geq-\frac{(p+q)}{c \rho_{0}} \quad \text { and } \quad \lambda_{\max }\left(\hat{B}_{l_{0}}^{0}\right)<-\frac{(p+q)}{c \rho_{0}},
$$

where $1 \leq l_{0} \leq l$. We project to select $l$ nodes and rearrange nodes of the complex network. Next, the value of $\lambda_{\max }\left(\hat{B}_{l}^{0}\right)$ is calculated, and when $l=l_{0}-1$ and $l=l_{0}$ satisfy (3.14), the number of pinned nodes is presented, that is, at least $l=l_{0}$.

\subsection{Finite-time synchronization problem of directed network}

Theorem 2 Suppose that Assumption 1 holds. Suppose that there exist positive constants $\varsigma_{1}, \varsigma_{2}, \varsigma_{3}, q$, and $d_{i}, i=1, \ldots, l$, such that

(1) $(p+q) I_{N}+c \rho_{0} \hat{B}^{0 s}-D<0$ ，

(2) $L_{2}-\frac{1-\psi_{1}}{2} k_{1}<0$,

(3) $a c_{\tau} \frac{\lambda_{\max }\left(\Gamma_{\tau}^{\mathrm{T}} \Gamma_{\tau}\right)}{2 \varsigma_{1}}+\frac{1}{2 \varsigma_{3}}-\frac{1-\psi_{2}}{2} k_{2}<0$,

(4) $a c_{\tau} \frac{\lambda_{\max }\left(\Gamma_{\tau}^{\mathrm{T}} \Gamma_{\tau}\right)}{2 \varsigma_{2}}-\frac{1-\psi_{3}}{2} k_{3}<0$,

(5) $t_{2}^{*}=t_{0}+\frac{\sqrt{2}}{k^{\prime}} V^{\frac{1}{2}}\left(t_{0}\right)$.

Theorem 2 is similar to Theorem 1, except condition (1). To construct symmetrical outer coupling matrices, we change the outer coupling matrix $B_{l}^{0}$ by $\hat{B}_{l}^{0^{s}}$. The proving process of a directed network is similar to that of an undirected network. Thus we omit it here. According to Lemma 4, the complex network (2.1) can achieve the synchronization in finite time

$$
t \leq t_{2}^{*}=t_{0}+\frac{\sqrt{2}}{k^{\prime}} V^{\frac{1}{2}}\left(t_{0}\right)
$$


Corollary 2 Comparing a directed network with an undirected network, the difference is in selection of pinned nodes. For a directed network, the degrees of one node are divided into two classes, out-degree and in-degree. Out-degree denotes that the quantity of a node point to other nodes, and in-degree represents that the quantity of other nodes points to the node. In this paper, let $\operatorname{Deg}_{\text {out }}(i)$ and $\operatorname{Deg}_{\text {in }}(i)$ denote, respectively, the in-degree and out-degree of node $i$, that is, $\operatorname{Deg}_{\text {out }}(i)=\sum_{j=1, j \neq i}^{N} b_{j i}^{0}$ and $\operatorname{Deg}_{\text {in }}(i)=\sum_{j=1, j \neq i}^{N} b_{i j}^{0}$. The difference between outdegree and in-degree is called the degree-difference of nodes expressed by $\operatorname{Deg}_{\text {diff }}(i)$. Hence, $\operatorname{Deg}_{\text {diff }}(i)=\operatorname{Deg}_{\text {out }}(i)-\operatorname{Deg}_{\text {in }}(i)$.

The pinned-nodes are selected in terms of the degree-difference of nodes. Rearrange the nodes in descending order according to their degree-differences and the nodes with same degree-differences rearrange in descending order according to their out-degrees.

For achieving the synchronization of a complex network in finite time, how many nodes need to be pinned at least? The method is similar to that for undirected networks. That is, condition $Q-D<0$ needs to be satisfied, and when $d$ is sufficiently large, $Q-D<0$ is equivalent to $Q_{l}<0$, right now, $Q_{l}=(p+q) I_{N-l}+c \rho_{0} \hat{B}_{l}^{0^{s}}$. Namely, $\left(p+a_{1}\right) I_{N-l}+c \rho_{0} \hat{B}_{l}^{0^{s}}<0$. We assume that the number of at least $l_{0}$ nodes need to be pinned so that accomplish condition $Q_{l}<0$, where $l_{0}$ satisfies

$$
\lambda_{\max }\left(\hat{B}_{l_{0}-1}^{0^{s}}\right) \geq-\frac{(p+q)}{c \rho_{0}} \quad \text { and } \quad \lambda_{\max }\left(\hat{B}_{l_{0}}^{0^{s}}\right)<-\frac{(p+q)}{c \rho_{0}} .
$$

\section{Numerical simulations}

In this section, some numerical simulations are given to demonstrate the effectiveness of the theoretical results for the finite-time synchronization. In the first place, a delayed neural network is selected as the isolated node in complex network (2.1):

$$
\dot{x}(t)=f\left(t, x(t), x\left(t-\tau_{1}(t)\right)\right)=A x(t)+C_{1} g_{1}(x(t))+C_{2} g_{2}\left(x\left(t-\tau_{1}\right)\right) .
$$

Let $x(t)=\left(x_{1}(t), x_{2}(t)\right)^{\mathrm{T}} \in \mathbf{R}^{2}, g_{1}(x)=g_{2}(x)=\left(\tanh \left(x_{1}\right), \tanh \left(x_{2}\right)\right)^{\mathrm{T}}, \tau_{1}=1$, and

$$
A=\left[\begin{array}{cc}
-1 & 0 \\
0 & -1
\end{array}\right], \quad C_{1}=\left[\begin{array}{cc}
3 & -0.2 \\
-4.5 & 5.5
\end{array}\right], \quad C_{2}=\left[\begin{array}{cc}
-2 & -0.1 \\
-0.3 & -5
\end{array}\right]
$$

Figure 1 shows that system (4.1) is chaotic.

Then, based on (2.4), the controlled synchronization state equation is described by

$$
\dot{s}(t)=A s(t)+C_{1} g_{1}(s(t))+C_{2} g_{2}\left(s\left(t-\tau_{1}\right)\right)+a c_{\tau} \Gamma_{\tau}\left(s\left(t-\tau_{2}(t)\right)-s\left(t-\tau_{3}(t)\right)\right) .
$$

Next, we consider an undirected network consisting of 100 nodes, and the model is described by

$$
\begin{aligned}
\dot{x}_{i}(t)= & A x(t)+C_{1} g_{1}(x(t))+C_{2} g_{2}\left(x\left(t-\tau_{1}\right)\right)+a c_{\tau} \Gamma_{\tau}\left(x_{i}\left(t-\tau_{2}(t)\right)-x_{i}\left(t-\tau_{3}(t)\right)\right) \\
& +c \sum_{j=1}^{N} b_{i j}^{0} \Gamma x_{j}(t)+c_{\tau} \sum_{j=1}^{N} b_{i j}^{1} \Gamma_{\tau} x_{j}\left(t-\tau_{2}(t)\right), \quad i=1,2, \ldots, 100,
\end{aligned}
$$




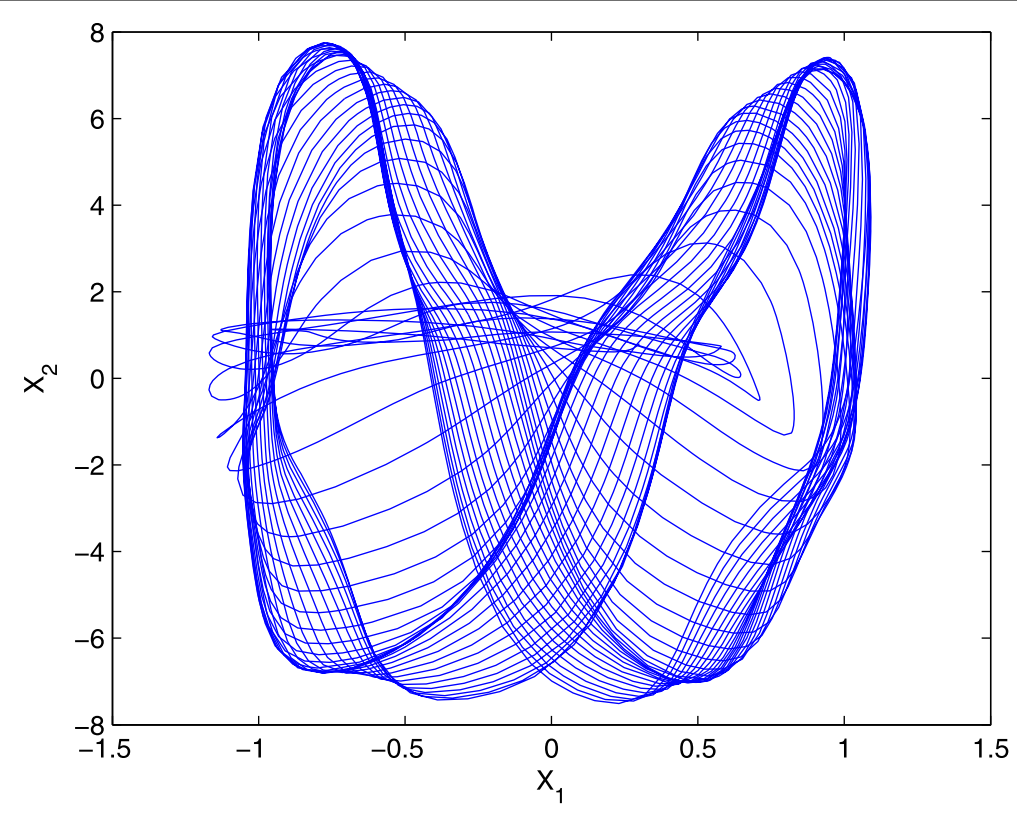

Figure 1 The chaotic state of system (4.1) with initial conditions $x_{1}(\tau)=0.3$ and $x_{2}(\tau)=0.8$, where $\tau \in[-1,0]$.

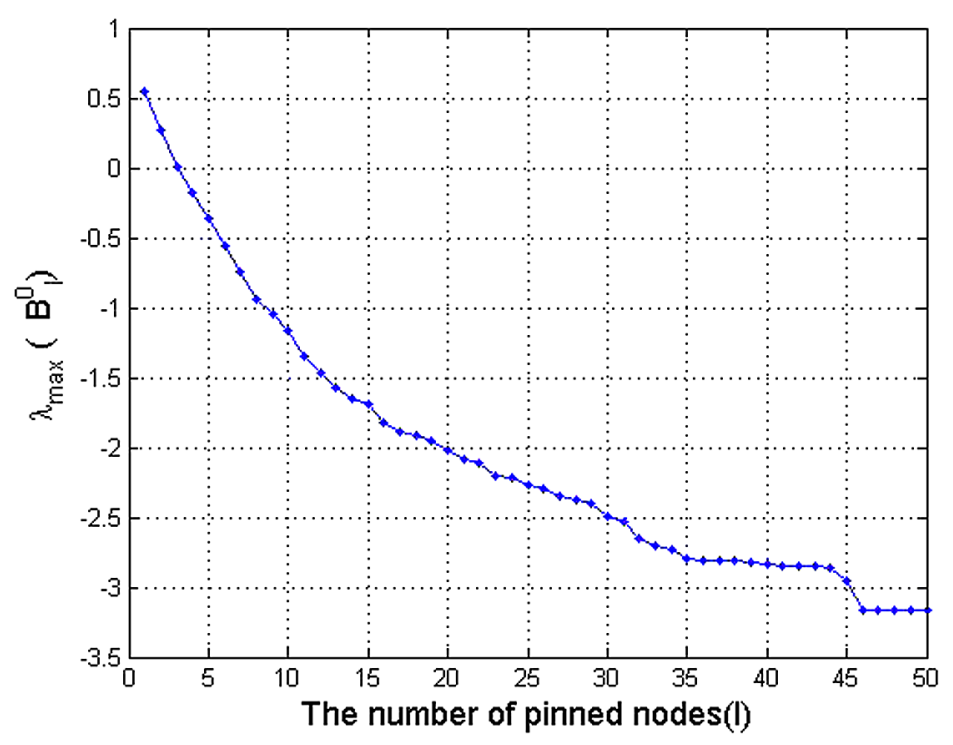

Figure 2 The relationship between the number of pinned-nodes and maximum eigenvalues.

where, $\Gamma=\operatorname{diag}(1.1,1), \Gamma_{\tau}=\operatorname{diag}(1,1), c=10, c_{\tau}=1, \tau_{2}(t)=e^{t} /\left(1+e^{t}\right)$, and $\tau_{3}(t)=$ $0.03|\cos (t)|$. According to the BA model [22], we can know that the coupling matrix $B^{0}$ is generated by the undirected network. Let $N=100$ and $B^{1}=0.10 \tilde{B}^{0}$, where $\tilde{B}^{0}=\left(\tilde{b}_{i j}^{0}\right)_{N \times N}=$ $\left(b_{i j}^{0} /\left(\sum_{j=1, j \neq i} b_{i j}^{0}\right)\right)_{N \times N}$. Next, by Corollary 1 , selecting some parameters values, we can obtain $\lambda_{\max }\left(\hat{B}_{l}^{0}\right)<-1.1861$.

We make use of the pinned-nodes selection method in the complex network (2.8), rearrange the network nodes, and choose $l$ from 1 to 50 . Then, depict $\lambda_{\max }\left(\hat{B}_{l}^{0}\right)$ in Figure 2 . 

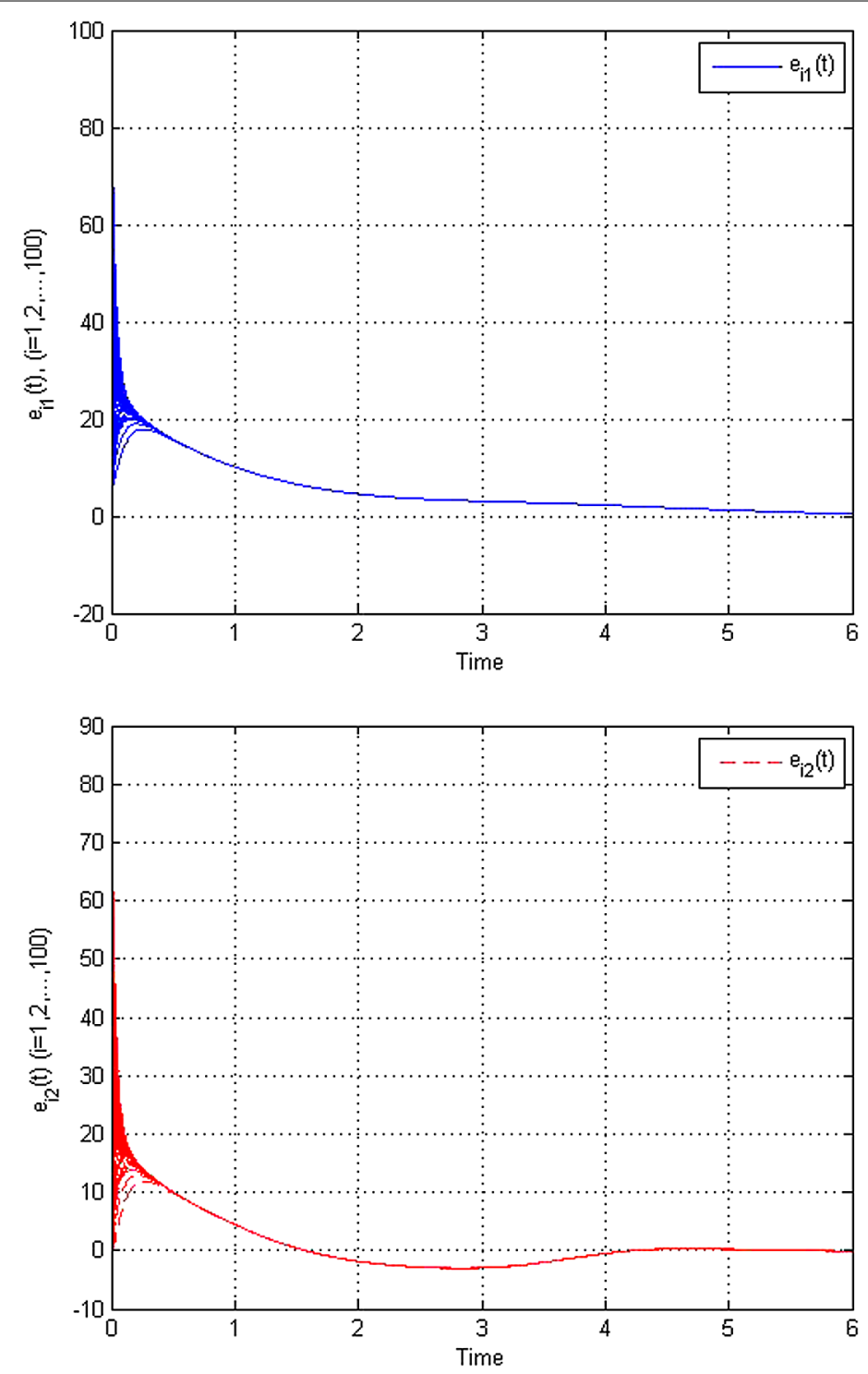

Figure 3 The curves of complex network synchronization errors.

From Figure 2 it is clear that the values of $\lambda_{\max }\left(\hat{B}_{l}^{0}\right)$ decrease along as the number of pinned-nodes $l$ increases. Especially, when $l=10$ and $l=11$, we get $\lambda_{\max }\left(\hat{B}_{10}^{0}\right)=-1.1688$ and $\lambda_{\max }\left(\hat{B}_{11}^{0}\right)=-1.3459$. Hence, we need only select the first $l_{0}=11$ rearranged nodes of network (4.3) as pinned nodes for the synchronization of complex network in finite-time. As shown in Figure 3, the synchronization errors $e_{i}(t)(1 \leq i \leq 100)$ tend to synchronize in finite time. Furthermore, the picture of the synchronization errors $e_{i}(t)$ in the directed network is similar to that of the undirected network and is omitted.

\section{Conclusion}

In this paper, we have investigated the finite-time synchronization problem of a delayed complex dynamical network via pinning control. This network model has two types of time-delays, internal delay and coupling delay, which make this network model closer to reality. Continuous nonsmooth control protocols are proposed from two aspects, an undi- 
rected network and a directed network. Sufficient conditions are given for the finite-time synchronization of a complex network by finite-time stability theory. Moreover, we have separately formulated the pinned-nodes schemes from undirected and directed networks.

The most significant aspect of the finite-time synchronization of complex networks is certainly the application value, that is to say, the synchronization system can be applied to various fields, such as cryptography communications, laser system, nervous system, traffic networks, internet, superconducting materials, and so on. The synchronization system is able to satisfied the exacting requirements of these applications on precision, velocity, quality, and costing. Finally, the numerical simulations have clearly verified the effectiveness of the developed pinning control to the finite-time synchronization of hybrid-coupled delayed dynamical networks.

Acknowledgements
This work was supported by the National Natural Science Foundation of China (No. 61305076) and the Funds for Basic
Creative Research of Department of Basic Science, Shijiazhuang Mechanical Engineering College (No. JCCX1601).

Competing interests

The authors declare that they have no competing interests.

Authors' contributions

Both authors contributed equally to the writing of this paper. Furthermore, both authors also read and carefully approved the final manuscript.

\section{Publisher's Note}

Springer Nature remains neutral with regard to jurisdictional claims in published maps and institutional affiliations.

Received: 11 July 2017 Accepted: 14 October 2017 Published online: 25 October 2017

References

1. Sun, X, Si, S: Complex Network Algorithms and Applications. National Defence Industry Press, Beijing (2015)

2. Lu, J, Liu, H, Chen, J: Synchronization in Complex Dynamical Network. Higher Education Press, Beijing (2016)

3. Xu, J: The synchronization of complex networks and its application based on pinning control. Master dissertation, Nanjing University of Posts and Telecommunications (2014)

4. Cai, S, Zhou, P, Liu, Z: Pinning synchronization of hybrid-coupled directed delayed dynamical network via intermittent control. Chaos 24, 033102 (2014)

5. Qin, J, Zheng, W, Gao, H: A study of synchronization of complex dynamical network model. In: Proceeding of International Symposium on Circuits and Systems, Brazil, pp. 1596-1598 (2011)

6. Yu, W, Chen, G, LV, J: On pinning synchronization of linearly coupled complex dynamical networks. Automatica 45, 429-435 (2009)

7. $\mathrm{Wu}, \mathrm{W}, \mathrm{Zhou}, \mathrm{W}, \mathrm{Chen}, \mathrm{T}$ : Cluster synchronization of linearly coupled complex networks under pinning control. IEEE Trans. Circuits Syst. I, Regul. Pap. 56, 829-839 (2009)

8. Guo, X, Li, J: A new synchronization algorithm for delayed complex dynamical networks via adaptive control approach. Commun. Nonlinear Sci. Numer. Simul. 17, 4395-4403 (2012)

9. Zheng, S, Wang, S, Dong, G, Bi, Q: Adaptive synchronization of two nonlinearly coupled complex dynamical networks with delayed coupling. Commun. Nonlinear Sci. Numer. Simul. 17, 284-291 (2012)

10. Mei, J, Jiang, $M, X u, W$, Wang, B: Finite-time synchronization control of complex dynamical networks with time delay. Commun. Nonlinear Sci. Numer. Simul. 18, 2462-2478 (2013)

11. Sun, $\mathrm{W}, \mathrm{Hu}, \mathrm{T}, \mathrm{Chen}, \mathrm{Z}, \mathrm{XiaO}, \mathrm{L}:$ Impulsive synchronization of a general nonlinear coupled complex network. Commun. Nonlinear Sci. Numer. Simul. 16, 4501-4507 (2011)

12. Lu, R, Yu, W, Lv, J, Xue, A: Synchronization on complex networks of networks. IEEE Trans. Neural Netw. Learn. Syst. 25, 2110-2118 (2014)

13. Yu, D, Bai, X, Ren, W, Kang, C: Finite-time synchronization control of complex networks. J. Jilin Univ. 32, 0423-0429 (2014)

14. Cortes, J: Finite-time convergent gradient flows with applications to network consensus. Automatica 42, 1993-2000 (2006)

15. Jin, $X, H e, Y$, Wang, D: Adaptive finite-time synchronization of a class of pinned and adjustable complex networks. Nonlinear Dyn. 85, 1393-1403 (2016)

16. $\mathrm{Xu}, \mathrm{Y}, \mathrm{Zhou}, \mathrm{W}$, Fang, J, Xie, C, Tong, D: Finite-time synchronization of the complex dynamical network with non-derivative and derivative coupling. Neurocomputing 173, 1356-1361 (2016)

17. Mei, J, Jiang, M, Wu, Z, Wang, X: Periodically intermittent controlling for finite-time synchronization of complex dynamical networks. Nonlinear Dyn. 79, 295-305 (2015)

18. Perry, BM, Michaelson, GV, Ballard, MA: On the statistical detection of clusters in undirected networks. Comput. Stat. Data Anal. 68, 170-189 (2013) 
19. $\mathrm{Ma}, \mathrm{Q}, \mathrm{Lu}, \mathrm{J}$ : Cluster synchronization for directed complex dynamical networks via pinning control. Neurocomputing 101, 354-360 (2013)

20. Wang, J, Feng, J, Xu, C, Zhao, Y, Feng, J: Pinning synchronization of nonlinearly coupled complex networks with time-varying delays using M-matrix strategies. Neurocomputing 177, 89-97 (2016)

21. Liang, $Y$, Wang, $X:$ A method of quickly calculating the number of pinning nodes on pinning synchronization in complex networks. Appl. Math. Comput. 246, 743-751 (2014)

22. Barabási, AL, Albert, R: Emergence of scaling in random networks. Science 286, 509-512 (1999)

23. Ning, $D, W u, X, L u, J, L v, J$ : Driving-based generalized synchronization in two-layer networks via pinning control. Chaos 25, 113104 (2015)

Submit your manuscript to a SpringerOpen ${ }^{\circ}$ journal and benefit from:

- Convenient online submission

- Rigorous peer review

- Open access: articles freely available online

- High visibility within the field

- Retaining the copyright to your article

Submit your next manuscript at $\gg$ springeropen.com 